PROCEEDINGS OF THE

AMERICAN MATHEMATICAL SOCIETY

Volume 132, Number 10, Pages 2917-2927

S 0002-9939(04)07442-8

Article electronically published on June 2, 2004

\title{
POLYNOMIALS GENERATED BY LINEAR OPERATORS
}

\author{
P. GALINDO, M. L. LOURENÇO, AND L. A. MORAES
}

(Communicated by Jonathan M. Borwein)

\begin{abstract}
We study the class of Banach algebra-valued $n$-homogeneous polynomials generated by the $n^{\text {th }}$ powers of linear operators. We compare it with the finite type polynomials. We introduce a topology $w_{E F}$ on $E$, similar to the weak topology, to clarify the features of these polynomials.
\end{abstract}

The finite type scalar-valued polynomials play an important role when dealing with algebras of holomorphic functions. For instance, the spectrum of the Fréchet algebra of entire functions of bounded type on a complex Banach space $E$ with the approximation property coincides with $E^{\prime \prime}$ if, and only if, the finite type polynomials are dense in the algebra [2]. When dealing with holomorphic mappings with values in a complex Banach algebra $F$, a new class of "finite type" polynomials arises: In 12] the subspace $\mathbb{P}_{f}\left({ }^{n} E, F\right)$ of all $n$-homogeneous polynomials generated by $n^{\text {th }}$ powers of linear operators was introduced. In this paper we study the relationship of this subspace with other commonly used classes of polynomials. Unless otherwise stated, the space of $n$-homogeneous polynomials $P\left({ }^{n} E, F\right)$ will be endowed with the norm topology. For uniform Banach algebras $F$ and Banach spaces $E$ with the approximation property, we prove that the closure of the $n$-homogeneous finite type polynomials $P_{f}\left({ }^{n} E, F\right)$ and the closure of $\mathbb{P}_{f}\left({ }^{n} E, F\right)$ coincide if, and only if, all linear operators from $E$ into $F$ are compact. We also show that it may happen that the closure of $\mathbb{P}_{f}\left({ }^{n} E, F\right)$ lies strictly between the closure of $P_{f}\left({ }^{n} E, F\right)$ and the whole space of polynomials $P\left({ }^{n} E, F\right)$.

We introduce a topology $w_{E F}$ on $E$, like the weak topology, which is the coarsest that makes continuous all elements in $\mathbb{P}_{f}\left({ }^{n} E, F\right) \forall n \in \mathbb{N}$. When $F=\mathbb{C}$, this topology coincides with the weak topology $w$ in $E$. It is known that the finite type scalar polynomials on $E$ are the weakly continuous ones. However, it may happen that a polynomial is $w_{E F}$-continuous but not in $\mathbb{P}_{f}\left({ }^{n} E, F\right)$ ([12]; see also section 2). It is shown that the Aron-Berner extension of $w_{E F}$-continuous polynomials is $w_{E^{\prime \prime} F^{\prime \prime}}$ continuous and also that it extends polynomials in $\mathbb{P}_{f}\left({ }^{n} E, F\right)$ to polynomials in $\mathbb{P}_{f}\left({ }^{n} E^{\prime \prime}, F^{\prime \prime}\right)$ where $F^{\prime \prime}$ carries the Arens product.

For background on polynomials on Banach spaces we refer to [6].

Received by the editors September 4, 2002.

2000 Mathematics Subject Classification. Primary 46G20.

Key words and phrases. $n$-homogeneous polynomials, linear operator, Arens product.

The first author was supported by CCInt-USP and FAPEMIG.

The second author was supported in part by agreement USP/UV and FAPESP.

The third author was supported in part by CNPq, Research Grant 300016/82-4 and PROAP/UFRJ. 


\section{Polynomials generated By linear operators}

Let $E$ be a complex Banach space, and let $n \in \mathbb{N}$. If $F$ is a complex Banach algebra with identity, let $\mathbb{P}_{f}\left({ }^{n} E, F\right)$ denote the space generated by $\left\{T^{n}: T \in\right.$ $L(E, F)\}$, where $T^{n}(x)=(T(x))^{n}$ for each $x \in E$. This space was introduced in $\left[12\right.$ where it is shown that $P_{f}\left({ }^{n} E, F\right) \subset \mathbb{P}_{f}\left({ }^{n} E, F\right)$. Recall that $P_{f}\left({ }^{n} E, F\right)=$ $P_{f}\left({ }^{n} E\right) \otimes F$, where $P_{f}\left({ }^{n} E\right)$ is the space generated by the $n^{t h}$ powers of elements in $E^{\prime}$. This section begins with the study of the relationship between the norm closures of $\mathbb{P}_{f}\left({ }^{n} E, F\right)$ and the finite type polynomials. Later we will check that the Aron-Berner extension of a polynomial in $\mathbb{P}_{f}\left({ }^{n} E, F\right)$ is the $n^{t h}$ power of the bitranspose of the generating linear operators.

Recall that given an arbitrary Banach space $(F,\|\|)$ one can define in $F$ a product $\odot$ and a norm $|\||||$ such that $(F,\||\||)$ is a commutative Banach algebra with identity. Indeed, let $e \in F$ with $\|e\|=1$ and consider $\varphi \in F^{\prime}$ such that $\varphi(e)=1$. Let $F_{1}=\operatorname{ker}(\varphi)$. Define $u \odot v:=a b e+(b x+a y)$ for $u=a e+x$ and $v=b e+y$ where $a, b \in \mathbb{C}$ and $x, y \in F_{1}$. Moreover, define $\||u\||=| a \mid+\| x \|$ for all $u=a e+x$ with $a \in \mathbb{C}$ and $x \in F_{1}$. It is easy to verify that \|\|$\|$ is equivalent to \|\| and that $(F,\|\| \|)$ endowed with the product $\odot$ is a commutative Banach algebra with identity. It is also clear that such a $\varphi$ turns out to be a multiplicative form.

If $E$ is a Banach space and $F$ is a Banach algebra, it is easy to check that $\varphi \circ P \in P_{f}\left({ }^{n} E\right)$ whenever $P \in \mathbb{P}_{f}\left({ }^{n} E, F\right)$ and $\varphi \in F^{\prime}$ is multiplicative.

Observe that $\overline{P_{f}\left({ }^{n} E\right)}=P\left({ }^{n} E\right)$ whenever $\overline{\mathbb{P}_{f}\left({ }^{n} E, F\right)}=P\left({ }^{n} E, F\right)$ for some Banach algebra $F$. Indeed, let $P \in P\left({ }^{n} E\right)$. Take a homomorphism $\varphi: F \longrightarrow \mathbb{C}$ and $e \in F$ such that $\varphi(e)=1$ and define $Q: E \longrightarrow F$ by $Q(x):=P(x) \cdot e \forall x \in E$. Clearly $P=\varphi \circ Q$ and $Q \in P\left({ }^{n} E, F\right)$. Since $\overline{\mathbb{P}_{f}\left({ }^{n} E, F\right)}=P\left({ }^{n} E, F\right)$ by hypothesis, there exists $\left(Q_{k}\right) \subset \mathbb{P}_{f}\left({ }^{n} E, F\right)$ such that $Q_{k} \stackrel{\|\|}{\longrightarrow} Q$ and so $\left\|\varphi \circ Q_{k}-\varphi \circ Q\right\| \leq$ $\|\varphi\| \cdot\left\|Q_{k}-Q\right\| \longrightarrow 0$. Since $\left(\varphi \circ Q_{k}\right) \subset P_{f}\left({ }^{n} E\right)$ and $\varphi \circ Q=P$, we have $P \in \overline{P_{f}\left({ }^{n} E\right)}$.

It is known that $\overline{P_{f}\left({ }^{n} E\right)}=P\left({ }^{n} E\right)$ does not imply $\overline{P_{f}\left({ }^{n} E, F\right)}=P\left({ }^{n} E, F\right)$ for every Banach space $F$. The same situation occurs for $\mathbb{P}_{f}\left({ }^{n} E, F\right)$, that is, it may happen that $\overline{\mathbb{P}_{f}\left({ }^{n} E, F\right)} \neq P\left({ }^{n} E, F\right)$ despite $\overline{P_{f}\left({ }^{n} E\right)}=P\left({ }^{n} E\right)$. We give some explanation for the reader's convenience. Indeed, take $E=\ell_{3}$ and $F=\ell_{2}$ endowed with the product $\odot$ introduced above. All $\mathbb{C}$-valued 2-homogeneous polynomials on $\ell_{3}$ are weakly uniformly continuous on bounded sets by Pitt's theorem, hence $\overline{P_{f}\left({ }^{2} \ell_{3}\right)}=P\left({ }^{2} \ell_{3}\right)$. On the other hand, since $L\left(\ell_{3}, \ell_{2}\right)=K\left(\ell_{3}, \ell_{2}\right)$, it follows from Proposition 2.5 in $\left[12\right.$ that $\overline{\mathbb{P}_{f}\left({ }^{2} \ell_{3}, \ell_{2}\right)}=\overline{P_{f}\left({ }^{2} \ell_{3}, \ell_{2}\right)}$. This shows that $\mathbb{P}_{f}\left({ }^{2} \ell_{3}, \ell_{2}\right)$ is not dense, since the polynomial $\left(x_{i}\right) \in \ell_{3} \mapsto\left(x_{i}^{2}\right) \in \ell_{2}$ is not a compact one.

Now we are going to establish conditions under which $\overline{P_{f}\left({ }^{n} E, F\right)}=\overline{P_{f}\left({ }^{n} E, F\right)}$. For this we require the following result.

Theorem 1.1. Let $E$ be a Banach space, and let $F$ be a uniform Banach algebra. If $T \in L(E, F)$ is such that $T^{n}, n \in \mathbb{N}$, is a (weakly) compact polynomial, then $T$ is a (resp. weakly) compact operator.

Proof. Since $F$ is a closed subspace of $\mathcal{C}(K)$ for some compact set $K$, it suffices to prove that $T$ is a (weakly) compact operator from $E$ into $\mathcal{C}(K)$.

Assume first that $T^{n}$ is compact. By the Ascoli Theorem it suffices to show that $T\left(B_{E}\right)$ is an equicontinuous subset of $\mathcal{C}(K)$. Let $u \in K$. If $T(x)(u)=0$ for all $x \in B_{E}$, given $\epsilon>0$ there exists a neighbourhood $U$ of $u$ in $K$ such that $\left|T^{n}(x)(t)\right|<$ 
$\epsilon^{n}$ for all $t \in U$ and for all $x \in B_{E}$ since $T^{n}\left(B_{E}\right)$ is clearly equicontinuous. So $|T(x)(t)-T(x)(u)| \leq \epsilon \forall t \in U$ and $\forall x \in B_{E}$.

Suppose now that there exists $a \in E$ such that $T(a)(u) \neq 0$. Without loss of generality, we may suppose that $T(a)(u)=1$. Given $\epsilon>0$ there exists a neighbourhood $W$ of $u$ such that $\left|T^{n-1}(a)(t)-1\right|<\frac{\epsilon}{2|| T \mid}$ for all $t \in W$ since $T^{n-1}(a) \in \mathcal{C}(K)$. Moreover, $T^{n-1}(a) T\left(B_{E}\right)$ is equicontinuous since $T^{n-1}(a) \cdot T$ is a compact operator from $E$ into $\mathcal{C}(K)$ by Proposition 3.4 of $[3$. Consequently, there exists a neighbourhood $V$ of $u$ in $K$ such that $V \subset W$ and

$$
\left|\left[T^{n-1}(a) \cdot T(x)\right](t)-T(x)(u)\right|<\frac{\epsilon}{2}
$$

for all $x \in B_{E}$ and for all $t \in V$. Therefore,

$$
\begin{aligned}
\mid T(x) & (t)-T(x)(u) \mid \\
& \leq\left|T(x)(t)-\left[T^{n-1}(a) \cdot T(x)\right](t)\right|+\left|\left[T^{n-1}(a) \cdot T(x)\right](t)-T(x)(u)\right| \\
& =|T(x)(t)| \cdot\left|1-T^{n-1}(a)(t)\right|+\left|\left[T^{n-1}(a) \cdot T(x)\right](t)-T(x)(u)\right| \\
& \leq \| T|| \frac{\epsilon}{2\|T\|}+\frac{\epsilon}{2}=\epsilon
\end{aligned}
$$

for all $x \in B_{E}$ and for all $t \in V$.

The argument for the weakly compact case is similar. According to [7, Theorem 14, p. 269, it suffices to show that $T\left(B_{E}\right)$ is quasi-equicontinuous, that is, to show that given $u \in K$ and a net $\left(u_{\alpha}\right)_{\alpha \in A} \subset K$ converging to $u$, for $\epsilon>0$ and $\alpha_{0} \in A$ there are a finite number of indices, $\alpha_{1}, \ldots, \alpha_{m} \in A$ with $\alpha_{i} \geq \alpha_{0}$ such that

$$
\min _{1 \leq i \leq m}\left|T(x)\left(u_{\alpha_{i}}\right)-T(x)(u)\right| \leq \epsilon \quad \forall x \in B_{E} .
$$

Since $T^{n}\left(B_{E}\right)$ is weakly relatively compact, it is quasi-equicontinuous; hence in case $T(x)(u)=0$ for all $x \in B_{E}$, we get, for $\epsilon>0$ and $\alpha_{0} \in A$, the existence of $\alpha_{1}, \ldots, \alpha_{m} \in A$ with $\alpha_{i} \geq \alpha_{0}$ such that

$$
\min _{1 \leq i \leq m}\left|T^{n}(x)\left(u_{\alpha_{i}}\right)\right|=\min _{1 \leq i \leq m}\left|T^{n}(x)\left(u_{\alpha_{i}}\right)-T^{n}(x)(u)\right| \leq \epsilon^{n} \quad \forall x \in B_{E},
$$

which is enough.

Suppose now that there exists $a \in E$ such that $T(a)(u)=1$. Given $\epsilon>0$ and $\alpha_{0} \in A$ the continuity of $T^{n-1}$ leads to $\alpha_{0}^{\prime}$ such that

$$
\left|1-T^{n-1}(a)\left(u_{\alpha}\right)\right|=\left|T^{n-1}(a)(u)-T^{n-1}(a)\left(u_{\alpha}\right)\right| \leq \frac{\epsilon}{2|| T||} \quad \forall \alpha \geq \alpha_{0}^{\prime} .
$$

By Theorem 3.2 in [14] the derivative mapping $n T^{n-1}(a) T: E \rightarrow \mathcal{C}(K)$ is a weakly compact mapping. In particular, $\left[T^{n-1}(a) T\right]\left(B_{E}\right)$ is a weakly relatively compact set and therefore $\alpha_{1}, \ldots, \alpha_{m} \in A$ with $\alpha_{i} \geq \alpha_{0}^{\prime}$ may be found such that

$$
\min _{1 \leq i \leq m}\left|T^{n-1}(a)\left(u_{\alpha_{i}}\right) \cdot T(x)\left(u_{\alpha_{i}}\right)-T^{n-1}(a)(u) \cdot T(x)(u)\right| \leq \frac{\epsilon}{2} \quad \forall x \in B_{E} .
$$

Then

$$
\begin{aligned}
& \left|T(x)\left(u_{\alpha_{i}}\right)-T(x)(u)\right| \\
& \leq\left|T(x)\left(u_{\alpha_{i}}\right)-\left[T^{n-1}(a) \cdot T(x)\right]\left(u_{\alpha_{i}}\right)\right|+\left|\left[T^{n-1}(a) \cdot T(x)\right]\left(u_{\alpha_{i}}\right)-T(x)(u)\right| \\
& =\left|T(x)\left(u_{\alpha_{i}}\right)\right| \cdot\left|1-T^{n-1}(a)\left(u_{\alpha_{i}}\right)\right|+\left|\left[T^{n-1}(a) \cdot T(x)\right]\left(u_{\alpha_{i}}\right)-T^{n-1}(a)(u) T(x)(u)\right| .
\end{aligned}
$$


Thus for all $x \in B_{E}$,

$$
\begin{aligned}
\min _{1 \leq i \leq m} & \left|T(x)\left(u_{\alpha_{i}}\right)-T(x)(u)\right| \\
\leq & \frac{\epsilon}{2}+\min _{1 \leq i \leq m}\left|T^{n-1}(a)\left(u_{\alpha_{i}}\right) \cdot T(x)\left(u_{\alpha_{i}}\right)-T^{n-1}(a)(u) \cdot T(x)(u)\right| \leq \epsilon .
\end{aligned}
$$

Corollary 1.2. Let $E$ be a Banach space such that $E^{\prime}$ has the approximation property, and let $F$ be a uniform Banach algebra. The following statements are equivalent:

(1) $L(E, F)=K(E, F)$.

(2) $\overline{P_{f}\left({ }^{n} E, F\right)}=\overline{\mathbb{P}_{f}\left({ }^{n} E, F\right)} \forall n \geq 1$.

(3) $\overline{P_{f}\left({ }^{n} E, F\right)}=\overline{\mathbb{P}_{f}\left({ }^{n} E, F\right)}$ for some $n \geq 1$.

Proof. (1) $\Rightarrow$ (2) Apply Proposition 2.5 of [12.

(3) $\Rightarrow$ (1) If $\overline{P_{f}\left({ }^{n} E, F\right)}=\overline{\mathbb{P}_{f}\left({ }^{n} E, F\right)}$, then every element of $\overline{\mathbb{P}_{f}\left({ }^{n} E, F\right)}$ is compact. Now, given $T \in L(E, F)$ we have that $T^{n}$ is a compact polynomial and so, by Theorem 1.1, $T$ is compact.

Under the assumptions of the above corollary the equivalent conditions mean also that $\overline{\mathbb{P}_{f}\left({ }^{n} E, F\right)}$ coincides with the space of all compact polynomials. Theorem 1.1 shows that all elements in $L(E, F)$ are weakly compact if, and only if, all elements in $\mathbb{P}_{f}\left({ }^{n} E, F\right)$ are weakly compact. It is tempting to ask whether in this case $\overline{\mathbb{P}_{f}\left({ }^{n} E, F\right)}$ coincides with the space of all weakly compact polynomials; however, the example quoted before Theorem 1.1 prevents such a coincidence.

Remark 1.3. Theorem 1.1 is not true if the Banach algebra $F$ is not uniform as the following example shows:

Consider $T: c_{0} \rightarrow L\left(\ell_{\infty}, \ell_{\infty}\right)$ defined by

$$
T(x)(y)=\left(0, x_{1} \cdot y_{1}, 0, x_{3} \cdot y_{3}, 0, \ldots, 0, x_{2 n+1} y_{2 n+1}, 0, \ldots\right)
$$

for all $x=\left(x_{i}\right) \in c_{0}$ and $y=\left(y_{i}\right) \in \ell_{\infty}$. It is clear that $T$ is a linear operator such that $T^{2}$ is compact since $T^{2} \equiv 0$. Nevertheless $T$ is not compact since the sequence $\left(T\left(e_{2 n+1}\right)\right)$ does not have convergent subsequences. Neither $T$ is weakly compact; otherwise the transpose mapping $T^{t}$ would be a weakly compact operator into the Schur space $\ell_{1}$, and hence $T^{t}$ would be compact.

Recall that a set $A \subset E$ is said to be a limited set if weak* null sequences in $E^{*}$ are uniformly convergent on $A$. The Josefson-Nissenzweig Theorem guarantees that the unit ball of any infinite-dimensional Banach space is not a limited set (see [6], p. 234).

Theorem 1.4. Let $E$ be a Grothendieck space having a quotient isomorphic to $\ell_{p}$. Then

$$
\overline{P_{f}\left({ }^{n} E, c\right)} \mp \overline{\mathbb{P}_{f}\left({ }^{n} E, c\right)} \varsubsetneqq P\left({ }^{n} E, c\right) \quad \forall n \geq p, \quad n \in \mathbb{N} .
$$

Proof. Since $E$ is a Grothendieck space, all elements in $L(E, c)$ are weakly compact. Recall that in a uniform algebra the product of two weakly relatively compact sets is a weakly relatively compact set. Then we have that every element in $\mathbb{P}_{f}\left({ }^{n} E, c\right)$ maps bounded sets into weakly relatively compact sets, and the same holds for all the elements in $\overline{\mathbb{P}_{f}\left({ }^{n} E, c\right)}$. 
Fix $n \geq p$. In order to prove that $\overline{\mathbb{P}_{f}\left({ }^{n} E, c\right)} \varsubsetneqq P\left({ }^{n} E, c\right)$ we define a continuous $n$-homogeneous polynomial $Q: \ell_{p} \longrightarrow c$ by

$$
Q(x)=\left(-\sum_{i=1}^{\infty} x_{i}^{n},(-1)^{2} \sum_{i=2}^{\infty} x_{i}^{n}, \ldots,(-1)^{k} \sum_{i=k}^{\infty} x_{i}^{n}, \ldots\right)
$$

for every $x=\left(x_{i}\right)_{i=1}^{\infty} \subset \ell_{p}$. We remark that $Q$ is not weakly compact since the sequence $\left(Q\left(e_{m}\right)\right)_{m=1}^{\infty} \subset Q\left(\overline{B_{\ell_{p}}}\right)$ does not have a weakly convergent subsequence. Now, if $q: E \rightarrow \ell_{p}$ is the quotient mapping, there exists $\lambda>0$ such that $\lambda Q\left(B_{\ell_{p}}\right) \subset$ $(Q \circ q)\left(B_{E}\right)$. Therefore, $Q \circ q$ is not weakly compact and $(Q \circ q) \in P\left({ }^{n} E, c\right) \backslash$ $\overline{\mathbb{P}_{f}\left({ }^{n} E, c\right)}$.

Since $c$ is a Banach algebra with identity, $P_{f}\left({ }^{n} E, c\right) \subset \mathbb{P}_{f}\left({ }^{n} E, c\right)([12$, Proposition 2.2). Furthermore, $\overline{P_{f}\left({ }^{n} E, c\right)} \varsubsetneqq \overline{\mathbb{P}_{f}\left({ }^{n} E, c\right)}$ because otherwise $\overline{\mathbb{P}_{f}\left({ }^{n} E, c\right)}=$ $\overline{P_{f}\left({ }^{n} E, c\right)}$ and then Corollary 1.2 would yield that $L(E, c)=K(E, c)$, which is not possible since there are noncompact operators from $E$ into $c$ because the unit ball of $E$ is not limited.

Corollary 1.5. Let $E$ be a Grothendieck space. If $\overline{\mathbb{P}_{f}\left({ }^{n} E, c\right)}=P\left({ }^{n} E, c\right)$ for some $n>1$, then $E$ is reflexive.

Proof. Suppose that $E$ is nonreflexive. By Proposition 1 of [8], $E$ contains a copy of $\ell_{1}$, and hence it has a quotient isomorphic to $\ell_{2}$. Therefore by Theorem 1.4 we have that $\overline{\mathbb{P}_{f}\left({ }^{n} E, c\right)} \varsubsetneqq P\left({ }^{n} E, c\right)$ for all $n \geq 2$, a contradiction.

The elements in $\mathbb{P}_{f}\left({ }^{n} E, F\right)$ behave in the most suitable way regarding the AronBerner extension to the bidual. We refer the reader to [5] for a survey of properties of the Arens extension to $F^{\prime \prime}$ of the product in $F$.

Proposition 1.6. The Aron-Berner extension of every $P \in \mathbb{P}_{f}\left({ }^{n} E, F\right)$ belongs to $\mathbb{P}_{f}\left({ }^{n} E^{\prime \prime}, F^{\prime \prime}\right)$ when $F^{\prime \prime}$ is considered as carrying the left Arens product.

Proof. We have to show that for any $L \in L(E, F), L^{\prime \prime n}$, the $n$th power of the double transpose $L^{\prime \prime}: E^{\prime \prime} \rightarrow F^{\prime \prime}$, coincides with the Aron-Berner extension of the polynomial $L^{n}$.

Recall that for a multilinear mapping $A \in L\left({ }^{n} E, F\right)$, its Aron-Berner extension $\tilde{A}$ and fixed $a_{1}, \ldots, a_{j-1} \in E$ and $a_{j+1}^{\prime \prime}, \ldots, a_{n}^{\prime \prime} \in E^{\prime \prime}$, the mapping

$$
x^{\prime \prime} \in E^{\prime \prime} \mapsto \tilde{A}\left(a_{1}, \ldots, a_{j-1}, x^{\prime \prime}, a_{j+1}^{\prime \prime}, \ldots, a_{n}^{\prime \prime}\right)
$$

is weak*-weak* continuous (see [6], 6.2).

Now, for $T_{1}, \ldots, T_{n} \in L(E, F)$ the $n$-linear mapping $A:=T_{1} \cdot \ldots \cdot T_{n}: E \times \ldots \times$ $E \rightarrow F$ given by $A\left(x_{1}, \ldots, x_{n}\right)=T_{1}\left(x_{1}\right) \cdot \ldots \cdot T_{n}\left(x_{n}\right)$ may be extended according to $\left(x_{1}^{\prime \prime}, \ldots, x_{n}^{\prime \prime}\right) \in E^{\prime \prime} \times \ldots \times E^{\prime \prime} \mapsto T_{1}^{\prime \prime}\left(x_{1}\right) \cdot \ldots \cdot T_{n}^{\prime \prime}\left(x_{n}\right) \in F^{\prime \prime}$. Since the transpose mappings are always weak*-weak* continuous and for the left Arens product the mappings $f^{\prime \prime} \in F^{\prime \prime} \mapsto f^{\prime \prime} \cdot g^{\prime \prime} \in F^{\prime \prime}$ and $f^{\prime \prime} \in F^{\prime \prime} \mapsto h \cdot f^{\prime \prime} \in F^{\prime \prime}$ are weak*-weak* continuous for fixed $g^{\prime \prime} \in F^{\prime \prime}$ and $h \in F$ respectively (see [5], p. 311), it follows that the mapping

$$
x^{\prime \prime} \in E^{\prime \prime} \mapsto T_{1}\left(a_{1}\right) \cdot \ldots \cdot T_{j-1}\left(a_{j-1}\right) \cdot T_{j}^{\prime \prime}\left(x^{\prime \prime}\right) \cdot T_{j+1}\left(a_{j+1}^{\prime \prime}\right) \cdot \ldots \cdot T_{n}^{\prime \prime}\left(a_{n}^{\prime \prime}\right)
$$

is also weak ${ }^{*}$-weak* continuous. Therefore by the weak ${ }^{*}$ density of $E$ in $E^{\prime \prime}$, we obtain that $\tilde{A}=T_{1}^{\prime \prime} \cdot \ldots \cdot T_{n}^{\prime \prime}$. 


\section{The $w_{E F}$ TOPOlOgy AND The $w_{E F}$-CONTINUOUS POLYNOMials}

Let $E$ and $F \neq\{0\}$ be complex normed spaces. For each $T \in L(E, F)$ we set $p_{T}(x):=\|T(x)\|$ for all $x \in E$. Then $p_{T}$ is a seminorm on $E$, and the family $\left\{p_{T}: T \in L(E, F)\right\}$ defines a locally convex topology $w_{E F}$ on $E$. This topology is the weakest topology on $E$ that makes continuous all $T \in L(E, F)$ and hence, all polynomials in $\mathbb{P}_{f}\left({ }^{n} E, F\right) \forall n \in \mathbb{N}$. We denote by $L_{w s c}(E, F)$ the subspace of all weakly sequentially continuous elements of $L(E, F)$.

It is easy to verify that the weak topology is coarser than the $w_{E F}$ topology, which in turn is also coarser than the norm topology and consequently, $w_{E F}$ is compatible with the dual pair $\left(E, E^{\prime}\right)$. Note that $w_{E F}=w$ in case $F=\mathbb{C}$, and $w_{E F}$ is the norm topology in case $E$ is a subspace of $F$. Later, Propositions 2.3, 2.4 and 2.5 show that these topologies can be different.

Remark 2.1. The relationship between different $w_{E F}$ topologies when varying the reference spaces may be summarized as follows: For fixed $E$, whenever $Y$ is a subspace of $Z$, then $w_{E Y} \preceq w_{E Z}$, and for fixed $Y$, whenever $E$ is a subspace of $F$, then $w_{F Y_{\left.\right|_{E}}} \preceq w_{E Y}$.

Proposition 2.2. Let $E$ and $F$ be Banach spaces, and let $T$ be a linear mapping from $E$ into $F$. Then $T$ is continuous if and only if $T:\left(E, w_{E F}\right) \longrightarrow(F, w)$ is continuous.

Proof. If $T:\left(E, w_{E F}\right) \longrightarrow(F, w)$ is continuous, $T\left(x_{n}\right) \stackrel{w}{\longrightarrow} T(x)$ whenever $x_{n} \stackrel{w_{E F}}{\longrightarrow}$ $x$. Now, if $\left(x_{n}, T\left(x_{n}\right)\right) \longrightarrow(x, y)$ in $E \times F$ we have $T\left(x_{n}\right) \stackrel{w}{\longrightarrow} T(x)$ since $w_{E F}$ is weaker than the norm topology and clearly $T\left(x_{n}\right) \stackrel{w}{\longrightarrow} y$. So, $T(x)=y$ and we have that $T: E \longrightarrow F$ is continuous by the Closed Graph Theorem.

The converse is clearly true since $w \preceq w_{E F}$.

Proposition 2.3. Let $E$ and $F$ be Banach spaces. The following statements are equivalent:

(1) $L(E, F)=L_{w s c}(E, F)$.

(2) Given any sequence $\left(x_{n}\right)_{n \in \mathbb{N}}$ in $E$ we have that $\left(x_{n}\right)_{n \in \mathbb{N}}$ converges to $x$ in $(E, w)$ if and only if $\left(x_{n}\right)_{n \in \mathbb{N}}$ converges to $x$ in $\left(E, w_{E F}\right)$.

(3) The weak topology $w$ and the $w_{E F}$ topology coincide on the weakly compact subsets of $E$.

Proof. The equivalence between (1) and (2) is clear. It is also clear that (3) implies (2).

$(2) \Rightarrow(3)$ Let $K$ be a weakly compact subset of $E$. It is enough to show that $\bar{A}^{w}=\bar{A}^{w_{E F}}$ for all $A \subset K$. Since $A \subset K$ is weakly relatively compact, given any $x \in \bar{A}^{w}$, there exists, by the Eberlein-Smulian Theorem, $\left(x_{n}\right)_{n \in \mathbb{N}} \subset A$ such that $x_{n} \stackrel{w}{\longrightarrow} x$. By using (2) we get $\bar{A}^{w} \subset \bar{A}^{w_{E F}}$, and the proof is complete since clearly $\bar{A}^{w_{E F}} \subset \bar{A}^{w}$.

To emphasize the different behaviour of the $w_{E F}$ topology and the norm or the weak topology, we point out, for instance, that the canonical embedding $\iota: E \rightarrow E^{\prime \prime}$ may not be $\left(w_{E F}-w_{E^{\prime \prime} F^{\prime \prime}}\right)$-continuous: just pick $E=\ell_{\infty}$ and $F=c_{0}$, and remark that $L(E, F)=L_{w s c}(E, F)$ since $E=\ell_{\infty}$ is a Grothendieck space and has the Dunford-Pettis property. Since any weakly convergent sequence $\left(x_{n}\right) \subset \ell_{\infty}$ is $w_{E F^{-}}$ convergent by Proposition 2.3, if $\iota: E \rightarrow E^{\prime \prime}$ were $\left(w_{E F}-w_{E^{\prime \prime} F^{\prime \prime}}\right)$-continuous, 
$\left(\iota\left(x_{n}\right)\right)$ would be $w_{E^{\prime \prime} F^{\prime \prime}}$-convergent. In particular, for the extension to $E^{\prime \prime}$ of the mapping $\iota: E \rightarrow \ell_{\infty}$, that is, the projection $\pi:\left(\ell_{\infty}\right)^{\prime \prime} \rightarrow \ell_{\infty}$, since $\pi \in L\left(E^{\prime \prime}, F^{\prime \prime}\right)$, we would obtain that $\left(x_{n}\right)=\left(\pi\left(\iota\left(x_{n}\right)\right)\right)$ norm converges in $F^{\prime \prime}=\ell_{\infty}$. Consequently, $\ell_{\infty}$ would be a Schur space.

Proposition 2.4. Let $E$ and $F$ be Banach spaces. The following statements are equivalent:

(1) $L(E, F)=K(E, F)$.

(2) Given any bounded net $\left(x_{\beta}\right)_{\beta \in I}$ in $E$ we have that $\left(x_{\beta}\right)_{\beta \in I}$ converges to $x$ in $(E, w)$ if, and only if, $\left(x_{\beta}\right)_{\beta \in I}$ converges to $x$ in $\left(E, w_{E F}\right)$.

(3) The weak topology $w$ and the $w_{E F}$ topology coincide on the bounded subsets of $E$.

Proof. The equivalence between (2) and (3) is clear.

$(1) \Rightarrow(2)$ Let $\left(x_{\beta}\right)_{\beta \in I}$ be a bounded net in $E$ such that $x_{\beta} \stackrel{w}{\longrightarrow} x$. Since $L(E, F)=K(E, F)$, every $T \in L(E, F)$ is weakly continuous on the bounded set $\left\{x_{\beta}: \beta \in I\right\} \cup\{x\}$ and consequently $T\left(x_{\beta}\right) \longrightarrow T(x)$ for all $T \in L(E, F)$. This means that $x_{\beta} \stackrel{w_{E F}}{\longrightarrow} x$. From this the result follows.

$(2) \Rightarrow(1)$ If (2) is true, by Proposition 2.3 we have that $L(E, F)=L_{w s c}(E, F)$. So, if $L(E, F) \neq K(E, F)$ there exists $T \in L_{w s c}(E, F)$ such that $T \notin K(E, F)$. Consequently, there exists a bounded subset $X$ of $E$ such that $\left.T\right|_{X}$ is not weakly continuous. So, there exists $x \in X$ and $\left(x_{\beta}\right)_{\beta \in I} \subset X$ such that $x_{\beta} \stackrel{w}{\longrightarrow} x$ but $T\left(x_{\beta}\right) \stackrel{\Perp \Perp}{\longrightarrow} T(x)$. This means that $x_{\beta} \stackrel{w_{E F}}{\longrightarrow} x$ despite $x_{\beta} \stackrel{w}{\longrightarrow} x$.

\section{Proposition 2.5.}

(1) If $E$ is a reflexive infinite-dimensional Banach space, then there exists a sequence $\left(x_{k}\right)_{k \in \mathbb{N}}$ in $E$ such that $x_{k} \stackrel{w_{E \ell_{1}}}{\longrightarrow} x$ and $x_{k} \stackrel{\|}{\longrightarrow} x$.

(2) If $L(E, F)=L_{w s c}(E, F)$ and $E$ is not a Schur space, then there exists a sequence $\left(x_{k}\right)_{k \in \mathbb{N}}$ in $E$ such that $x_{k} \stackrel{w_{E F}}{\longrightarrow} x$ and $x_{k} \Downarrow \| x$.

Proof. (1) Since $E$ is reflexive, there exists $\left(x_{k}\right)_{k \in \mathbb{N}} \subset E$ such that $x_{k} \stackrel{w}{\longrightarrow} x$ and $x_{k} \stackrel{\text { 竖 }}{\longrightarrow} x$. On the other hand, $x_{k} \stackrel{w_{E \ell_{1}}}{\longrightarrow} x$ since $L\left(E, \ell_{1}\right)=L_{w s c}\left(E, \ell_{1}\right)$.

The proof of (2) is similar.

Proposition 2.6. Let $E$ and $F$ be Banach spaces. Consider the following statements:

(1) $L(E, F)$ is reflexive.

(2) $E$ is reflexive and $L(E, F)=K(E, F)$.

(3) $\bar{B}_{E}$ is $w_{E F}$-compact.

Then $(2) \Leftrightarrow(3)$. Also, (2) $\Rightarrow(1)$ when $F$ is a reflexive space. If, furthermore, either $E$ or $F$ has the compact approximation property, then $(1) \Rightarrow(2)$.

Proof. $(2) \Rightarrow(3)$ Follows from Proposition 2.4.

$(3) \Rightarrow(2)$ The reflexivity of $E$ is a consequence of $w \preceq w_{E F}$. Since every $T \in$ $L(E, F)$ is $w_{E F}$-continuous, $T\left(B_{E}\right)$ is relatively compact in $F$.

If $F$ is reflexive, $(2) \Rightarrow(1)$ is just part of Theorem 1 of [11]. 
$(1) \Rightarrow(2)$ Under the condition (1), both $E$ and $F$ are reflexive spaces since $E^{*}$ and $F$ are isomorphic to complemented subspaces of $L(E, F)$. Then, if $E$ or $F$ has the compact approximation property, we apply Theorem 2.1 of [13] to complete the proof.

Recall that a completely regular Hausdorff space $X$ is a $k$-space when a subset $G$ of $X$ is open if and only if its intersection with each compact subset $K$ of $X$ is open in $K$. The space $\left(E, w_{E F}\right)$ is completely regular since it is a locally convex space.

If $F=\mathbb{C}$, it is known that $\left(E, w_{E F}\right)$ is a $k$-space if and only if $\operatorname{dim} E<\infty$ (since $w_{E F}$ coincides with the weak topology). In general we have:

Proposition 2.7. Let $E$ and $F$ be complex Banach spaces such that $\left(E, w_{E F}\right)$ is a $k$-space. If $L(E, F)=K(E, F)$ (respectively, $L(E, F)=L_{w s c}(E, F)$ ), then $E$ is reflexive (resp., reflexive or Schur).

Proof. Assume $L(E, F)=K(E, F)$. By Proposition 2.4, the weak topology and the $w_{E F}$ topology coincide on the bounded subsets of $E$. So, if $b w$ denotes the finest topology in $E$ that induces the weak topology on every bounded subset of $E$, it is obvious that $w_{E F} \preceq b w$ and that a subset $K$ of $E$ is $w_{E F}$-compact if and only if it is bw-compact. So, given a bw-closed subset $C$ of $E$ we have that $C \cap K$ is $w_{E F}$-closed for every $w_{E F^{-}}$-compact $K$ and, by our hypothesis, $C$ is $w_{E F^{-}}$-closed. This implies that $w_{E F}=b w$; so $b w$ is a locally convex topology, and this has been shown to imply that $E$ is reflexive (see 10 , Theorem 3.7).

In the case $L(E, F)=L_{w s c}(E, F)$, we replace in the above argument bw by $k w$, the finest topology agreeing with the weak topology on weakly compact sets which was introduced in [9], to come up with $w_{E F}=k w$, which in turn implies $E$ to be a reflexive or Schur space by Theorem 2.9 in $[9$.

Let $P_{w_{E F}}\left({ }^{n} E, F\right)$ be the space of all $w_{E F}$-continuous $n$-homogeneous polynomials from $E$ into $F$. The set $P_{w_{E F}}(E, F)=\bigoplus_{n=0}^{\infty} P_{w_{E F}}\left({ }^{n} E, F\right)$ of all $w_{E F}$-continuous polynomials from $E$ into $F$ is a complex algebra, since for given $Q \in P_{w_{E F}}\left({ }^{n} E, F\right)$ and $P \in P_{w_{E F}}\left({ }^{m} E, F\right)$, the polynomial $P Q$ is also $w_{E F}$-continuous. Indeed, given $\epsilon>0$ there exist $T_{1}, T_{2}, \ldots, T_{k} \in L(E, F)$ such that $\|Q(x)\|<\sqrt{\epsilon}$ and $\|P(x)\|<\sqrt{\epsilon}$ whenever $\sup _{1 \leq i \leq k}\left\|T_{i}(x)\right\|<1$. So $\sup _{1 \leq i \leq k}\left\|T_{i}(x)\right\|<1 \Rightarrow\|Q(x) P(x)\| \leq$ $\|Q(x)\|\|\mid\|(x) \|<\epsilon$. Therefore, $P Q$ is $w_{E F}$-continuous since $w_{E F}$ is a locally convex topology.

Now we may construct $w_{E F}$-continuous polynomials just by multiplying continuous functionals on $E$ by $w_{E F}$-continuous polynomials from $E$ into $F$.

If $E$ is a subspace of $F$, we have $P_{w_{E F}}\left({ }^{n} E, F\right)=P\left({ }^{n} E, F\right)$, and if $F=\mathbb{C}$, we have $P_{w_{E \mathbb{C}}}\left({ }^{n} E\right)=P_{w}\left({ }^{n} E\right)$ where $P_{w}\left({ }^{n} E\right)$ denotes the space of all weakly continuous $n$-homogeneous polynomials. It is well known that $P_{f}\left({ }^{n} E\right)=P_{w}\left({ }^{n} E\right)$.

Clearly, $\mathbb{P}_{f}\left({ }^{n} E, F\right) \subset P_{w_{E F}}\left({ }^{n} E, F\right)$. If $F=\mathbb{C}$, we have $\mathbb{P}_{f}\left({ }^{n} E\right)=P_{w}\left({ }^{n} E\right)$, and it is natural to ask whether $\mathbb{P}_{f}\left({ }^{n} E, F\right)=P_{w_{E F}}\left({ }^{n} E, F\right)$ for a Banach algebra $F$. This is not true in general. For instance, in case $E=c_{0}$ and $F=\ell_{\infty}$, since $c_{0} \subset \ell_{\infty}$, every element of $P\left({ }^{n} c_{0}, \ell_{\infty}\right)$ is $w_{c_{0} \ell_{\infty}}$-continuous. If $P: c_{0} \longrightarrow \ell_{\infty}$ is defined by $P\left(\left(x_{k}\right)_{k \in \mathbb{N}}\right)=a \cdot\left(\sum_{k=0}^{\infty} \frac{1}{2^{k}} x_{k}^{n}\right)$ where $a=\left(a_{j}\right)_{j \in \mathbb{N}} \in c_{0}, a \neq 0$, it is clear that $P \in P\left({ }^{n} c_{0}, \ell_{\infty}\right)$ and $P \notin \mathbb{P}_{f}\left({ }^{n} c_{0}, \ell_{\infty}\right)$ (cf. Example 2.3 of [12]). 
Proposition 2.8. Let $E$ be a complex Banach space. The following statements are equivalent:

(1) $E$ is a finite-dimensional space.

(2) $\mathbb{P}_{f}\left({ }^{n} E, F\right)=P_{w_{E F}}\left({ }^{n} E, F\right)$ for every $n \in \mathbb{N}$ and for every complex Banach algebra with identity $F$.

(3) $\mathbb{P}_{f}\left({ }^{n} E, F\right)=P_{w_{E F}}\left({ }^{n} E, F\right)$ for some $n \in \mathbb{N}$ and for every complex Banach algebra with identity $F$.

Proof. If $E$ is a finite-dimensional space, the $w_{E F}$ topology coincides with the norm topology on $E$, and by using Proposition 2.3 of [12] we get $(1) \Rightarrow(2)$. It is obvious that $(2) \Rightarrow(3)$.

Let us show that $(3) \Rightarrow(1)$. Fix $e \in E$ with $\|e\|=1$ and $\varphi \in E^{\prime}$ such that $\varphi(e)=1$, and consider the complex Banach algebra $F=(E, \odot)$ defined at the very beginning of $\S 1$ for the given Banach space $E$. In this case, the $w_{E F}$ topology coincides with the norm topology on $E$, and so $P_{w_{E F}}\left({ }^{n} E, F\right)=P\left({ }^{n} E, F\right)$ for all $n \in \mathbb{N}$. If $E$ is an infinite-dimensional space, then there exists a biorthogonal system $\left\{x_{k}, f_{k}: k \in \mathbb{N}\right\}$ such that $\left\{x_{k}: k \in \mathbb{N}\right\} \subset E,\left\{f_{k}: k \in \mathbb{N}\right\} \subset E^{\prime}$ and $f_{k}\left(x_{j}\right)=\delta_{k j}$. It is clear that

$$
Q(x):=\sum_{k=1}^{\infty} \frac{1}{2^{k}\left\|f_{k}\right\|} f_{k}^{n}(x), \quad x \in E,
$$

defines a continuous $n$-homogeneous polynomial in $E$. To complete the proof it is enough to show that the continuous $n$-homogeneous polynomial $P: E \longrightarrow F$ defined by $P(x):=Q(x) \cdot e$ for all $x \in E$ does not belong to $\mathbb{P}_{f}\left({ }^{n} E, F\right)$. Indeed, if $P \in \mathbb{P}_{f}\left({ }^{n} E, F\right)$, we have $Q \in P_{f}\left({ }^{n} E\right)$ by Remark 2.8 since $\varphi \circ P=Q$. Then $Q=\sum_{i=1}^{m} \psi_{i}^{n}$ where $\left\{\psi_{1}, \ldots, \psi_{m}\right\} \subset E^{\prime}$, and then for each $x \in E$ we have

$$
d Q(x)=\sum_{i=1}^{m} n \psi_{i}^{n-1}(x) \psi=\sum_{k=1}^{\infty} \frac{n}{2^{k}|| f_{k} \|} f_{k}^{n-1}(x) f_{k} .
$$

So, for every $j \in \mathbb{N}, d Q\left(x_{j}\right)=\frac{n}{2^{3} \mid f f_{j} \|} \cdot f_{j}=\sum_{i=1}^{m} n \psi_{i}^{n-1}\left(x_{j}\right) \psi_{i}$, and this means that $\left\{f_{k}: k \in \mathbb{N}\right\}$ is a subset of the finite-dimensional subspace of $E^{\prime}$ spanned by $\left\{\psi_{1}, \ldots, \psi_{m}\right\}$. This contradicts the linear independence of the set $\left\{f_{k}: k \in \mathbb{N}\right\}$.

Proposition 1.6 implies that the Aron-Berner extension of polynomials in

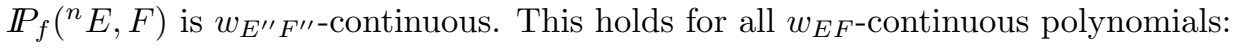

Proposition 2.9. The Aron-Berner extension of a $w_{E F}$-continuous polynomial is $w_{E^{\prime \prime} F^{\prime \prime} \text {-continuous. }}$

Proof. By the polarization formula the multilinear mapping associated to a $w_{E F^{-}}$ continuous polynomial is also $w_{E F}$-continuous. Let $A \in L\left({ }^{n} E, F\right)$ be $w_{E F}$-continuous. Then there are $T_{i, j} \in L(E, F), i=1, \ldots, n, j=1, \ldots, j(i)$, such that

$$
\left\|A\left(x_{1}, \ldots, x_{n}\right)\right\| \leq \sup _{1 \leq j \leq j(1)}\left\|T_{1, j}\left(x_{1}\right)\right\| \cdot \ldots \cdot \sup _{1 \leq j \leq j(n)}\left\|T_{n, j}\left(x_{n}\right)\right\| .
$$


Since the norm in $F^{\prime \prime}$ is weak* lower semicontinuous, each of the functions $x^{\prime \prime} \in$ $E^{\prime \prime} \mapsto \sup _{1 \leq j \leq j(i)}\left\|T_{i, j}^{\prime \prime}\left(x^{\prime \prime}\right)\right\|$ is weak ${ }^{*}$ lower semicontinuous on $E^{\prime \prime}$. Therefore, if $\left(z_{\alpha}\right) \subset E^{\prime \prime}$ weak* ${ }^{*}$ converges to $x^{\prime \prime} \in E^{\prime \prime}$, then by ([4], IV, §6, Prop. 4)

$$
\underline{\lim }_{\alpha} \sup _{1 \leq j \leq j(i)}\left\|T_{i, j}^{\prime \prime}\left(z_{\alpha}\right)\right\|=\sup _{1 \leq j \leq j(i)}\left\|T_{i, j}^{\prime \prime}\left(x^{\prime \prime}\right)\right\|, \quad i=1, \ldots, n .
$$

Since for fixed $f$ in the unit ball of $F^{\prime}$, and $a_{1}, \ldots, a_{k-1} \in E$ and $a_{k+1}^{\prime \prime}, \ldots, a_{n}^{\prime \prime} \in$ $E^{\prime \prime}$, the mapping

$$
x^{\prime \prime} \in E^{\prime \prime} \mapsto\left|f \circ \tilde{A}\left(a_{1}, \ldots, a_{k-1}, x^{\prime \prime}, a_{k+1}^{\prime \prime}, \ldots, a_{n}^{\prime \prime}\right)\right|
$$

is weak ${ }^{*}$ continuous on $E^{\prime \prime}$, we can prove that for a net $\left(x_{\alpha}\right) \subset E$ weak ${ }^{*}$ convergent to $x^{\prime \prime} \in E^{\prime \prime}$,

$$
\begin{aligned}
\left|f \circ \tilde{A}\left(a_{1}, \ldots, a_{k-1}, x^{\prime \prime}, a_{k+1}^{\prime \prime}, \ldots, a_{n}^{\prime \prime}\right)\right|=\lim _{\alpha}\left|f \circ \tilde{A}\left(a_{1}, \ldots, a_{k-1}, x_{\alpha}, a_{k+1}^{\prime \prime}, \ldots, a_{n}^{\prime \prime}\right)\right| \\
\quad \leq \sup _{1 \leq j \leq j(1)}\left\|T_{1, j}\left(a_{1}\right)\right\| \cdot \ldots \cdot \underline{\lim }_{\alpha} \sup _{1 \leq j \leq j(k)}\left\|T_{k, j}\left(x_{\alpha}\right)\right\| \cdot \ldots \cdot \sup _{1 \leq j \leq j(n)}\left\|T_{n, j}^{\prime \prime}\left(a_{n}^{\prime \prime}\right)\right\| \\
\quad=\sup _{1 \leq j \leq j(1)}\left\|T_{1, j}\left(a_{1}\right)\right\| \cdot \ldots \cdot \sup _{1 \leq j \leq j(k)}\left\|T_{k, j}^{\prime \prime}\left(x^{\prime \prime}\right)\right\| \cdot \ldots \cdot \sup _{1 \leq j \leq j(n)}\left\|T_{n, j}^{\prime \prime}\left(a_{n}^{\prime \prime}\right)\right\| .
\end{aligned}
$$

To check this we begin with the last variable and go backwards until the first one, and, finally, we obtain

$$
\left|f \circ \tilde{A}\left(a_{1}^{\prime \prime}, \ldots, a_{n}^{\prime \prime}\right)\right| \leq \sup _{1 \leq j \leq j(1)}\left\|T_{1, j}^{\prime \prime}\left(a_{1}^{\prime \prime}\right)\right\| \cdot \ldots \sup _{1 \leq j \leq j(n)}\left\|T_{n, j}^{\prime \prime}\left(a_{n}^{\prime \prime}\right)\right\| .
$$

This shows the $w_{E^{\prime \prime} F^{\prime \prime}}$-continuity of $\tilde{A}$.

\section{ACKNOWLEDGEMENTS}

Part of this paper was done during a São Paulo-Valencia exchange visit. The final version of it was written on the occasion of the 55을 SBA-Uberlândia. The authors gratefully thank Prof. Geraldo Botelho of Universidade Federal de Uberlândia for the support and hospitality. Also, the valuable referee's comments are appreciated.

\section{REFERENCES}

[1] R.M. Aron and P. Berner, A Hahn-Banach theorem for analytic mappings, Bull. Soc. Math. France 106 (1978), 3-24. MR 80e:46029

[2] R.M. Aron, B.J. Cole and T.W. Gamelin, Weak-star continuous analytic functions, Canad. J. Math. 47 (4) (1995), 673-683. MR 96d:46060

[3] R.M. Aron and M. Schottenloher, Compact holomorphic mappings on Banach spaces and the approximation property, J. Functional Anal. 21 (1976), 7-30. MR 53:6323

[4] N. Bourbaki, Éléments de mathématique. Topologie Générale, Hermann, Paris (1971). MR 50:11111

[5] J. Duncan and S.A.R. Hosseinium, The second dual of a Banach algebra, Proc. Roy. Soc. Edinburgh 84A (1979), 309-325. MR 81f:46057

[6] S. Dineen, Complex Analysis on Infinite Dimensional Spaces, Springer (1999). MR 2001a:46043

[7] N. Dunford and J. Schwartz, Linear Operators, Part I, Interscience (1958). MR 22:8302

[8] M. González and J. Gutiérrez, Polynomial Grothendieck properties, Glasgow Math. J. 37 (1995), 211-219. MR 96h:46025

[9] M. González and J. Gutiérrez, The compact weak topology on a Banach space, Proc. Roy. Soc. Edinburgh 120A (1992), 367-379. MR 93c:46022

[10] J. Gómez, On local convexity of bounded weak topologies on Banach spaces, Pacific J. Math. 110 (1984), 71-76. MR 85a:46014

[11] J. R. Holub, Reflexivity of $L(E, F)$, Proc. Amer. Math. Soc. 39 (1) (1973), 175-177. MR 47:3956 
[12] M. L. Lourenço and L. A. Moraes, A class of polynomials from Banach spaces into Banach algebras, Publ. Res. Inst. Math. Sci., Kyoto Univ. 37 (2001), 521-529. MR 2002k:46110

[13] J. Mujica, Reflexive spaces of homogeneous polynomials, Bull. Polish Acad. of Sci. 49 (3) (2001), 211-222. MR 2002i:46019

[14] R. Ryan, Weakly compact holomorphic mappings on Banach spaces, Pacific J. Math. 131 (1988), 179-190 MR 89a:46103

Departamento de Análisis Matemático, Facultad de Matemáticas, Universidad de Valencia 46.100, Burjasot-Valencia, Spain

E-mail address: Pablo.Galindo@uv.es

Departamento de Matemática, Instituto de Matemática e Estatística, Universidade de São Paulo, Caixa Postal 66281 - CeP : 05315-970, São Paulo, Brazil

E-mail address: mllouren@ime.usp.br

Instituto de Matemática, Universidade Federal do Rio de Janeiro, CP 68530 - CeP: 21945-970, Rio DE JANEIRo, BRAZIL

E-mail address: luiza@im.ufrj.br 